\title{
Desarrollo de un sistema de control inteligente basado en redes neuronales aplicado al proceso de pasteurización de lácteos
}

\section{Development of an intelligent control system based on neural networks applied to the dairy pasteurization process}

Adrián Alejandro Aldaz Vargas. ${ }^{1}$, Lando Stephen Ocaña Pañora. ${ }^{2}$, Janeth Ileana Arias Guadalupe. ${ }^{3}$ \& Jonatahn Paúl Campos Castelo. ${ }^{4}$

\begin{abstract}
.
DOI: https://doi.org/10.33262/concienciadigital.v4i2.2.1736

The pasteurization process requires the incorporation of technology in its pasteurization methods to improve its production. The objective of this system is to implement a pasteurization process of a milk plant, for this purpose the Matlab 2014 tool was used for the central control of the system considering the necessary conditions of the process. The pasteurizer control has a sensor and its actuators whose function is constant throughout the process which send signals through a data acquisition card and interpreted in Matlab 2014 and with the implementation of a human machine interface (HMI) through the Guide application. The pasteurization process initially uses raw milk at room temperature of $18^{\circ} \mathrm{C}$ according to NORMA NTE INEN 9:2012 (INEN, 2021), will be poured into the kettle to avoid waste, then begins the pasteurization process from the HMI of Matlab 2014 where the operator will choose the parameters necessary for its operation. For the

\footnotetext{
${ }^{1}$ Universidad Nacional de Chimborazo, Riobamba, Ecuador, aaldaz@unach.edu.ec, ORCID: 0000-00016290-0539

${ }^{2}$ Universidad de las Fuerzas Armadas “ESPE”, Latacunga, Ecuador, 1socana@espe.edu.ec, ORCID: 00000002-4748-5282

${ }^{3}$ Universidad Carlos III de Madrid, Madrid, España, jaariasg@ pa.uc3m.es, ORCID: 0000-00015540-1395

${ }^{4}$ Instituto Superior Tecnológico "Juan de Velasco", Riobamba, Ecuador. paulcamposjuandevelasco@gmail.com, ORCID: 0000-0002-8243-451X
} 
pasteurization process, the slow and open pasteurization method (VAT) is considered, which consists of heating large volumes of milk in 3 stages: heating, pasteurization, and cooling to finish the process.

Keywords: pasteurization, neural networks, intelligent control, VAT.

\section{Resumen.}

El proceso de pasteurización requiere la incorporación de tecnología en sus métodos de pasteurización con el fin de mejorar su producción. El objetivo de este sistema es implantar un proceso de pasteurización de una planta de leche, para esto se utilizó la herramienta Matlab 2014 utilizada para el control central del sistema tomando en cuenta las condiciones necesarias del proceso. El control del pasteurizador cuenta con un sensor y sus actuadores cuya función es constante en todo el proceso los cuales envían señales por una tarjeta de adquisición de datos e interpretados en Matlab 2014 y con implementación de una interfaz hombre maquina (HMI) mediante la aplicación Guide. El proceso de pasteurización utiliza inicialmente la leche cruda a temperatura ambiente de $18^{\circ} \mathrm{C}$ según la norma NORMA NTE INEN 9:2012 (INEN, 2021), será vertida en la marmita evitando su desperdicio, posteriormente se da comienzo al proceso de pasteurizado desde el HMI de Matlab 2014 donde el operador escogerá los parámetros necesarios para su funcionamiento. Para el proceso de pasteurizado se toma en cuenta el método de pasteurización lento y abierto (VAT) el cual consiste en calentar grandes volúmenes de leche en 3 etapas: calentamiento, pasteurización y enfriamiento para finalizar el proceso.

Palabras claves: pasteurización, redes neuronales, control inteligente, VAT.

\section{Introducción.}

La pasteurización se ha establecido con éxito en el contexto mundial como el método más utilizado para erradicar virus, hongos y patógenos de la leche en los países desarrollados y en desarrollo, Con la pasteurización se logra reducir los microorganismos presentes en el producto lácteo sin que esto afecte a las características propias del alimento. (Interempresas, 2018).

\section{Pasteurización de la leche}

Desde su origen la pasteurización es relacionada con la leche, este proceso ayuda a la eliminación de microorganismos que crecen en un rango de temperatura ambiente de $25^{\circ} \mathrm{C}$ a $37^{\circ} \mathrm{C}$, razón por la cual se evita su almacenamiento a esas temperaturas pasado el proceso de pasteurización. Con el paso de los años se han desarrollado varias técnicas para la eliminación de la mayoría de las agentes patógeno y evitar eliminar los nutrientes de la leche, varias técnicas generalmente son aplicadas bajo el punto de ebullición del agua en su mayoría. Además, disminuye la flora asociada, lo cual prolonga la vida útil 
del producto sin alterar su composición química y sus características organolépticas. (Tortora, Funke, \& Case, 2007).

\section{Proceso VAT}

Uno de los primeros métodos de pasteurización utilizado en la industria láctea es el proceso VAT que consiste en calentar volúmenes grandes de leche en un estanco a un rango de temperatura de $62^{\circ} \mathrm{C}$ a $65^{\circ} \mathrm{C}$ por un lapso de 30 minutos y posteriormente se debe pasar mucho tiempo para continuar con el proceso de envasado del producto veces más de 24 horas. (Tipán \& Flores, 2018)

\section{Proceso HTST}

El proceso High Temperature Short Time (HTST), consiste en someter un alimento a una temperatura próxima a $\operatorname{los} 79^{\circ} \mathrm{C}$ en un intervalo de tiempo de 15 segundos, la ventaja de este método es la rapidez en volúmenes grandes, permite eliminar los microorganismos patógenos, mediante la aplicación de alta temperatura durante un corto período de tiempo. (INOXPA, 2021).

\section{Proceso UHT}

El proceso Ultra High Temperature (UHT), consiste en someter un alimento a una temperatura próxima de $138^{\circ} \mathrm{C}$ en un intervalo de por lo menos 2 segundos, esto permite una degradación mínima del alimento para conservar los nutrientes y propiedades alimenticias, este proceso está dedicado a fabricar productos de larga duración no refrigerados, se consigue una eliminación de todos los microrganismos del producto tratado y sus formas de resistencia, consiguiendo así su esterilización. (IMPROLAC, 2021).

"La pasteurización es aquella operación para destruir por efecto del calor los microorganismos patógenos y en su mayoría de los gérmenes restantes, con fines higiénicos o de conservación, conservando al máximo las características físicas, bioquímicas y organolépticas del alimento. Dicha pasteurización permite la conservación en un tiempo determinado, basándose en las leyes de destrucción térmica de los microorganismos, estas leyes toman en consideración básicamente el número de microorganismos presentes, la temperatura en la que tiene lugar el proceso y el tiempo durante el que se mantiene dicha temperatura" (Gösta, 2003).

La industria láctea de Ecuador ha implementado recientemente nuevas políticas destinadas a mejorar los medios de vida, como cambiar la matriz de producción para permitir que los pequeños productores integren nuevas tecnologías para aumentar la producción. Esta política del estado permite el uso de nueva tecnología para ampliar el área de investigación para que el país se convierta en un productor de productos lácteos refinados. (Ministerio de Agricultura y Ganadería, 2020).

Los procesos industriales modernos, requieren un funcionamiento eficiente de los sistemas de control en una amplia gama de condiciones de funcionamiento en varios 
entornos hostiles; Donde se originan los sistemas de control inteligente que son basados en control borroso o fuzzy, control con redes neuronales, y control con algoritmos genéticos, con la finalidad de comandar mediante inteligencia artificial donde se permite emular características de comportamiento humano como son: la fácil adaptación, aprendizaje y planeación con grandes datos.

Dado que es un sistema de control de temperatura, se decidió desarrollar un sistema de control sensorial basado en la red neuronal para mejorar el sistema de control normal y apoyar la adquisición de nuevos métodos de aprendizaje y técnicas de investigación. Esta tecnología de control inteligente ha sido seleccionada para administrar una amplia variedad de datos para un mejor monitoreo. Una Red Neuronal Artificial (RNA) se basa en datos de aprendizaje, pruebas y entrenamiento, facilitando cambios en la práctica, es un modelo inspirado en como funciona el cerebro humano, conformado por nodos conocidos como neuronas artificiales conectadas entre sí. Estas señales van conectadas desde las entradas hasta posteriormente generar señales de salida. (ATRIA INNOVATION, 2021).

Un requisito para lograr generar redes neuronales es una etapa previa de entrenamiento en el cual se ingresa datos o información disponible del sistema en conjunto con una etapa de validación para comparar el aprendizaje de la red neuronal con otro grupo de datos disponibles.

Según Mendieta, Olarte, y González (2012), en un sistema real, la no linealidad reduce la eficiencia del sistema de fabricación. Por tanto, las estrategias de gestión técnica son muy eficaces para fortalecer el control. Los controles son más robustos y se pueden ajustar con diferentes valores. Las redes neuronales en los sistemas de control han sido probadas y usadas de una manera satisfactoria en los procesos no lineales como inyectores de plástico, intercambiadores de calor, robótica y visión artificial, etc.

La falta de artículos científicos en el Ecuador acerca del uso de redes neuronales en la industria es evidente. Sin embargo, el tema de investigación se puede aplicar en distintas áreas de desarrollo del proyecto. Esta técnica puede resolver problemas comunes que afectan de manera directa a la calidad de la leche y a la salud de los consumidores. El análisis, diseño e implementación de este sistema de control inteligente se basó en redes neuronales aplicado al proceso de pasteurización de leche con el método VAT con el objetivo de automatizar este proceso.

Se debe tomar en cuenta que la leche debe cumplir ciertas normas de higiene, para esto previamente pasara por una preparación bajo normas de asepsia previo al vaciado de un tanque pasteurizador. El método de control inteligente con base en redes neuronales nos permite un mejor comportamiento en el sistema no lineal.

Una Red Neuronal Artificial (RNA), usa como conjunto de entrada una serie de datos muy significativos para identificar patrones específicos o cierta regularidad de datos para conseguir una construcción aproximada del problema, culminado el entrenamiento de la 
red se podrá usar daros distintos a los iniciales sin degradación del rendimiento. (Palma \& Márin, 2011).

Se pretende mejorar el comportamiento de este sistema disminuyendo el tiempo de respuesta en comparación de los sistemas de control tradicionales y mejorando la estabilidad. De igual manera, se busca compensar las desventajas de la computación, la falta adaptabilidad y existencia de imprecisión e incertidumbre del control tradicional.

\section{Metodología.}

\section{Diseño e implementación}

Para el sistema de control inteligente con base en redes neuronales para el proceso de pasteurización de leche se utilizó el método heurístico con base en procesos empíricos y experimentales para el diseño e implementación del tablero de control, elementos, etc. Se tomó en cuenta las variables en cada etapa del proceso.

Las variables a medir son la temperatura, y velocidad del motor como se muestra en la tabla 1, se tomó una comparativa entre dos tratamientos distintos como son: el termómetro de alcohol y valor arrojado por el sensor de temperatura DS18B20, para el arranque del motor con distintas cargas dentro de la marmita se toma en cuenta la velocidad.

Tabla 1. Variables de sistema

\begin{tabular}{|c|c|}
\hline Variables del sistema & Consideraciones \\
\hline Temperatura & $\begin{array}{l}\text { - } \text { Rangos de temperatura de exposición de la leche. } \\
\text { - } \quad \text { Valores medidos mediante el sensor de temperatura } \\
\text { - } \quad \text { Registro de temperatura para el proceso } \\
\text { - Obtención de una mejor homogenización } \\
\text { - Estabilización de la homogenización para evitar el } \\
\text { - } \quad \text { Evite de la proteína de la leche } \\
\text { - }\end{array}$ \\
\hline Tiempo & $\begin{array}{l}\text { - Velocidad de homogenización y temperatura } \\
\text { dependen del tiempo } \\
\text { - Por medio del tiempo se escoge los datos brindados } \\
\text { por el sensor y velocidad de homogenización }\end{array}$ \\
\hline
\end{tabular}

Fuente: Elaboración propia de los autores.

\section{Sistema de control}

Se tomó en consideración la implementación de un sistema de control en lazo cerrado, para lo cual se describirá cada una de sus etapas.

\section{Sistema de control en lazo cerrado}

El sistema de control en lazo cerrado del proceso de pasteurización de leche consta de 4 etapas principales: 
- Primera etapa: Elección del controlador.

- Segunda etapa: Actuadores utilizados en el proceso.

- Tercera etapa: Planta u objetos a controlar como pasteurizador VAT o marmita.

- Cuarta etapa: Retroalimentación del sistema entre temperatura real y la deseada.

Figura 1. Diagrama de bloque de la temperatura del sensor DS18B20 del proceso de pasteurización de leche.

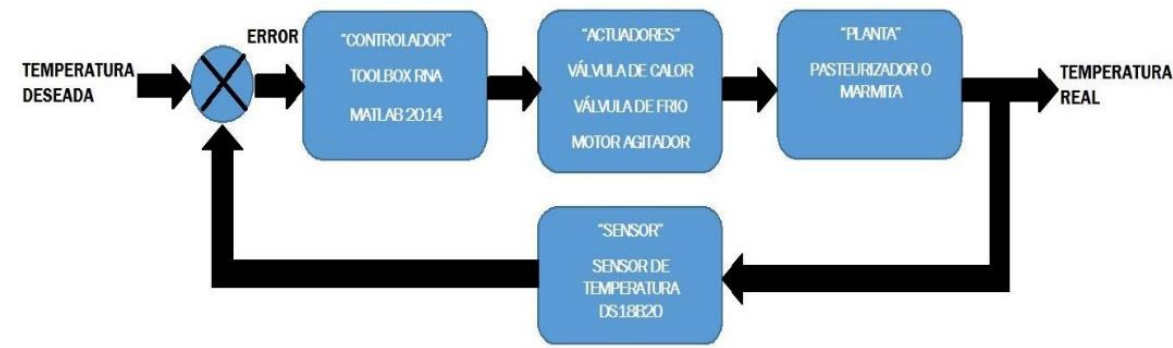

Fuente: Elaboración propia de los autores.

El diagrama de bloques (fig. 1), representa un sistema de control en lazo cerrado en el cual el valor de entrada Set Point el cual será la temperatura deseada, se tiene un error que es la diferencia entre la temperatura real y la deseada, la información pasa al controlador realizado en el toolbox RNA de Matlab 2014, siguiendo a los actuadores como son la válvula de calor, frio y el motor agitador para llegar a la planta de pasteurizado (marmita) y llegando a obtener una temperatura real sin antes realizar una retroalimentación.

En la figura 2 se aprecia el diagrama de bloques o esquemático del sistema de control de temperatura en lazo cerrado del pasteurizador o marmita.

Figura 2. Diagrama de bloque del sistema de control en lazo cerrado de temperatura del proceso de pasteurización de leche en la marmita (VAT).

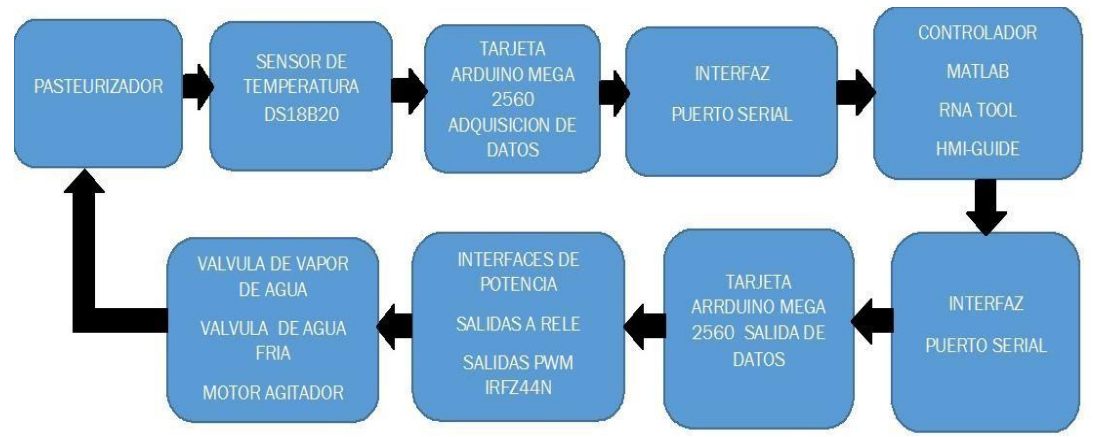

Fuente: Elaboración propia de los autores.

En el diagrama de bloques del proceso de pasteurización de leche se tiene que en la primera etapa realiza la lectura por el sensor DS18B20, en la segunda etapa se realiza una 
adquisición de datos del sensor de temperatura en el Arduino Mega 2560. En la tercera y cuarta etapa se tiene la interfaz de comunicación por puerto serie USB entre el Arduino como tarjeta de adquisición de datos y un controlador, este controlador es realizado en Toolbox RNA y HMI Guide como herramientas del software MATLAB.

Realizado el HMI todas las secuencias de pasos para el proceso de pasteurización los valores de salida de datos son enviadas por puerto serial a la tarjeta Arduino Mega 2560, estos datos de salida son valores lógicos 1 o 0 , el encendido o apagado de las electroválvulas y un valor de PWM con valores de 0 a 255 bits para activar un motor agitador. Los actuadores son activados por estos valores los cuales se acoplan al sistema de control en lazo cerrado por medio de interfaces de potencia usadas como acople de activación de relés autoprotegidos.

\section{Proceso de pasteurización de la leche}

El método VAT o abierto se utiliza para elaboración de diferentes productos lácteos, los procesos son de pasteurización, homogenización y enfriamiento. El operario tiene una Interfaz Hombre Maquina (HMI) en la cual puede escoger el tipo de producto lácteo para la pasteurización, la velocidad del agitador para la homogenización y el tiempo requerido en minutos. Al iniciar la HMI se acciona un breaker general en el tablero de control, el sistema abrirá una electroválvula de vapor de agua donde conducirá vapor hacia la marmita, el proceso de calentamiento se realiza hasta que alcance una temperatura máxima de pasteurización.

Para iniciar el motor agitador se debe activar un switch, el cual comienza a la homogenización mediante las aspas, una vez alcanzada la temperatura máxima de pasteurización la electroválvula de vapor es desconectada y comienza el tiempo de pasteurización y homogenización, el sistema hace una comparativa entre la temperatura actual es mayor que la temperatura mínima de pasteurización, en caso de ser cierto el sistema abre la válvula de agua fría y puede visualizarse en la HMI. Una vez alcanzado el valor mínimo de pasteurización se activa la electroválvula en conjunto con una alarma piloto y se podrá monitorear desde el tablero de control como en la HMI. Se presentarán los valores de temperatura, velocidad de homogenización y cambios en el proceso.

Figura 3. Proceso de pasteurización

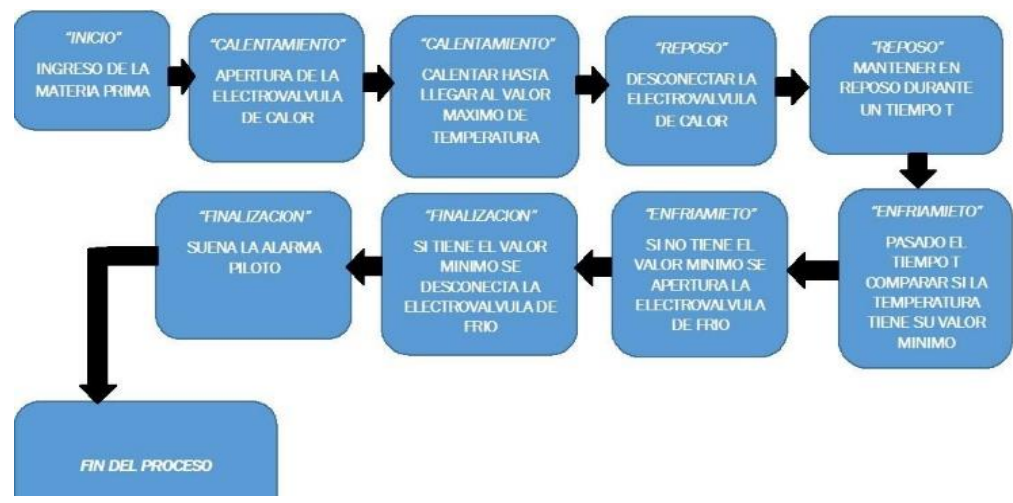

Fuente: Elaboración propia de los autores. 


\section{Control Inteligente}

El controlador neuronal implementado en Matlab 2014 ejecuta las acciones completas del sistema el cual interpreta los datos recibidos de la tarjeta de adquisición de datos, se tomó en cuenta la herramienta Toolbox de RNA incluida en Matlab 2014 para una red de backpropagation feedforward realizada con función de rendimiento MSE y un algoritmo de entrenamiento L-M.

\section{Entrenamiento de las Redes Neuronales Artificiales}

Se tomó en cuenta la red feedforward backpropagation incluida en el Toolbox RNA de Matlab 2014, inicialmente en la creación de la RNA se ingresa los datos de entrada/salida en forma de matrices en el script de Matlab 2014, se toma en cuenta dos RNA una de subida y otra de bajada por una repetición de datos en el calentamiento y enfriamiento se debe separar para evitar una incongruencia. El comando newff ayuda con la creación de la RNA en el cual se debe especificar ciertos valores mínimos y máximos de entrada, capas de entrada, capas ocultas, capas de salida y la función de activación.

Para el entrenamiento de la red neuronal se implementó un algoritmo de entrenamiento trainlm para la formación de red en función a los valores de entrada y salida en funciones de creación de red como newff entre otras, este comando actualiza los pesos y sesgo de la función de activación con el algoritmo L-M (Levenberg-Marquardt).

El algoritmo L-M es recomendable para procesos de entrenamiento con supervisión, en esta red fueron probados en sistemas no lineales en la robótica y procesos de rendimiento MSE. Se utilizó el comando sim para simular los valores de las RNA.

\section{Control de modulación por ancho de pulso (PWM)}

El control de modulación por ancho de pulso PWM se diseño para variar la velocidad de homogenización del motor agitador, este control describe los valores PWM que entrega la tarjeta controladora Arduino Mega 2560 con el rango de 0 a 255 bits PWM, se debe tomar en cuenta los porcentajes de ciclo de trabajo y voltaje.

Tabla 2. Control PWM

\begin{tabular}{ccccc}
\hline $\begin{array}{c}\text { PWM de } \\
\text { Arduino }\end{array}$ & $\begin{array}{c}\text { Amplitud } \\
\text { (VDC) }\end{array}$ & $\begin{array}{c}\text { Ciclo de } \\
\text { trabajo (\%) }\end{array}$ & $\begin{array}{c}\text { Numero de bits } \\
\text { de trabajo (bits) }\end{array}$ & $\begin{array}{c}\text { Valores óptimos para } \\
\text { homogenización }\end{array}$ \\
\hline 1 & $0-1,25$ & $0-25$ & $0-64$ & No \\
2 & $1,25-2,5$ & $25-50$ & $64-127$ & Adecuado \\
3 & $2,5-3,75$ & $50-75$ & $127-191$ & $\mathrm{Si}$ \\
4 & $3,75-5$ & $75-100$ & $191-255$ & $\mathrm{Si}$ \\
\hline
\end{tabular}

Fuente: Elaboración propia de los autores. 
Los valores del PWM son utilizados para el motor agitador acoplados con un IRFZ44N el cual esta diseñado para soportar altos amperajes en conjunto con un disipador ubicado en el tablero de control. (Hardware Libre, 2021).

Tabla 3. Características IRFZ44N

\begin{tabular}{cccccc}
\hline Características & $\begin{array}{c}\text { Voltaje de } \\
\text { ruptura } \\
\text { (VDC) }\end{array}$ & $\begin{array}{c}\text { Voltaje } \\
\text { Umbral } \\
\text { (VDC) }\end{array}$ & $\begin{array}{c}\text { Resistencia } \\
\text { de estado } \\
\text { ON }(\mathrm{m} \Omega)\end{array}$ & $\begin{array}{c}\text { Corriente } \\
(\mathrm{A})\end{array}$ & $\begin{array}{c}\text { Temperatura } \\
\left({ }^{\circ} \mathrm{C}\right)\end{array}$ \\
\hline IRFZ44N & 55 & 10 & 17,5 & 49 & 175 \\
\hline
\end{tabular}

Fuente: Elaboración propia de los autores

\section{Interfaz Hombre Maquina (HMI) del proceso}

La HMI consta de 8 etapas o secciones importantes, fue realizado en la herramienta Guide de Matlab 2014, está conectada de manera directa a una pantalla con la tarjeta de adquisición de datos Arduino lo cual nos ayuda con un entorno amigable.

Figura 4. Interfaz Hombre Máquina (HMI)

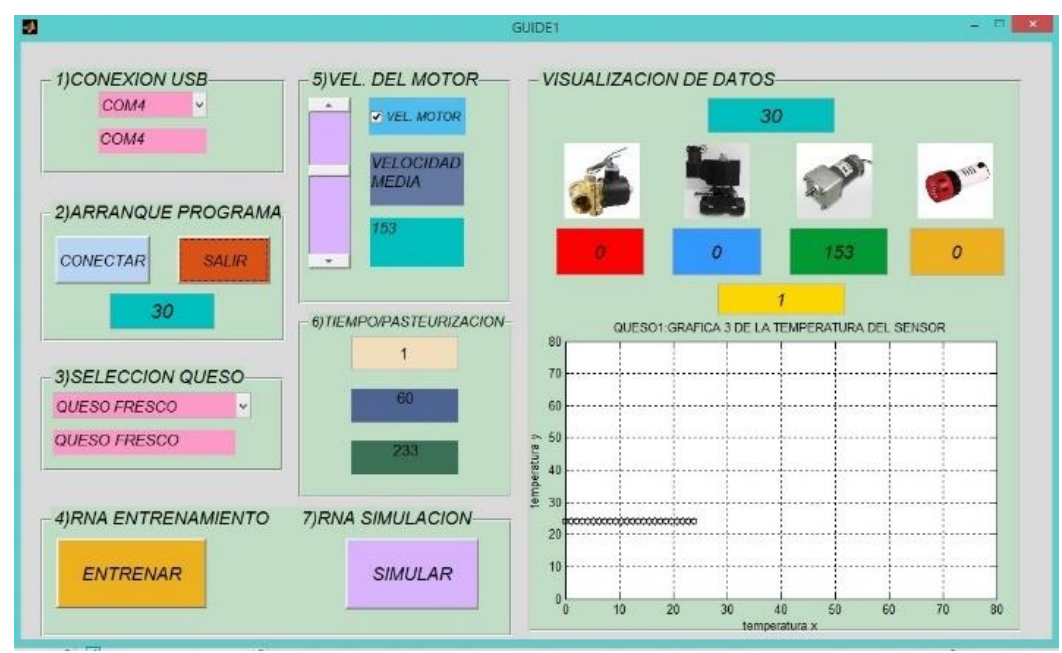

Fuente: Elaboración propia de los autores.

\section{Secciones del programa}

1. Se utiliza una comunicación por USB, para esta sección se habilitan dos botones, el botón Conectar para el arranque de la HMI del sistema, para salir del HMI se tiene el botón Salir, se puede visualizar el valor de la temperatura inicial.

2. El botón entrenar permite realizar un entrenamiento de las RNA de subida y bajada.

3. Una slider permite variar la velocidad del motor en conjunto con un checkbox, se podrá visualizar la velocidad del motor y su valor numérico de bits en PWM.

4. El tiempo lo puede ingresar en un edit-text en unidad de minutos no fraccionario. Una vez alcanzado el tiempo el programa sigue con la ejecución. 
5. El botón simular dará valores de entrada y salida a las redes neuronales, previo a la simulación se deben ingresar los valores de PWM y tiempo de pasteurización.

6. Para la visualización de datos se toma en cuenta un correcto monitoreo de sistema, se muestra el valor de la temperatura después de la simulación de la RNA.

7. En la programación del sistema se detalla las partes del sistema con sus respectivas líneas de código.

8. Muestra los valores de los actuadores " 0 " o " 1 " y una gráfica en tiempo real de la temperatura del sistema.

\section{Programación de la tarjeta de adquisición de datos Arduino Mega 2560}

Se utilizó como base el código de entradas digitales: Analog and Digital Input and Output Server for MATLAB (HaHn, 2014), este código ayudo a la adaptación del sensor a la tarjeta de adquisición de datos Arduino Mega 2560.

\section{Entorno de programación.}

El entorno de programación de Arduino brinda funciones básicas, se utilizaron las siguientes:

- Void setup (): La funsion setup() contiene la declaración de las variables, comunicación que se utilizara en el programa.

- Void loop (): la funsion $\operatorname{loop}()$ se ejecuta posterior a la setup (), esta función genera un bucle de lectura y escritura de datos de entradas y salidas de programa.

Figura 5. Programación para la lectura del sensor DS18B20 en Arduino 2560

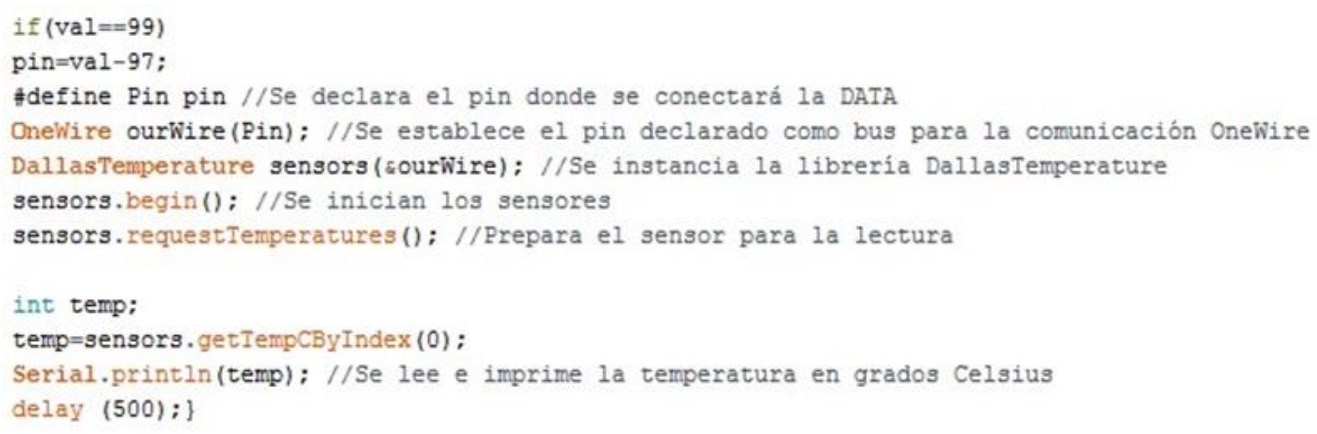

Fuente: Elaboración propia de los autores.

Se declara el pin 2 para la captación de datos del sensor DS18B20 en comunicación OneWire. Para este apartado se deben instalar las librerías OneWire y DallasTemperature para tener un valor de captado y de inicio del sensor.

El valor del sensor se almacena en una variable temp que mostrará el valor en grados centígrados $\left({ }^{\circ} \mathrm{C}\right)$ por puerto serial con un delay de 500 milisegundos en tiempo real. Se puede utilizar una velocidad de transmisión de datos de hasta 115200 entre el Arduino y Matlab 2014. 


\section{Resultados.}

Para medir los resultados de las pruebas realizadas al sistema y detallar las adecuaciones para que el proceso culmine de forma exitosa se utilizó el método inductivo-deductivo, científico-heurístico y comparativo. Las pruebas realizadas para un mejor rendimiento del proceso de pasteurización, pruebas de temperatura y mejora del tiempo de pasteurización se rigen bajo normas NTE INEN.

\section{Temperatura.}

El sensor DS18B20, permite realizar optimas mediciones de temperatura en el rango de $0^{\circ} \mathrm{C}$ a $85^{\circ} \mathrm{C}$ suficiente para el proceso cuya temperatura máxima llegará a $65^{\circ} \mathrm{C}$, este sensor puede realizar mediciones con mucha o poca leche en el pasteurizador, en un día de producción se tomó 20 lecturas durante el tiempo de pasteurización.

Tabla 4. Comparación temperaturas HMI sensor DS18B20 vs Termómetro de Alcohol

\begin{tabular}{|c|c|c|}
\hline Lectura & $\begin{array}{l}\text { Temperatura }\left({ }^{\circ} \mathrm{C}\right) \\
\text { tomado desde el HMI } \\
\text { sensor ds18b20 }\end{array}$ & $\begin{array}{c}\text { Temperatura }\left({ }^{\circ} \mathrm{C}\right) \text { tomado } \\
\text { desde el termómetro de } \\
\text { Alcohol }\end{array}$ \\
\hline 1 & 10 & 11 \\
\hline 2 & 12 & 12 \\
\hline 3 & 13 & 12 \\
\hline 4 & 13 & 13 \\
\hline 5 & 20 & 21 \\
\hline 6 & 22 & 22 \\
\hline 7 & 24 & 25 \\
\hline 8 & 25 & 27 \\
\hline 9 & 30 & 32 \\
\hline 10 & 33 & 33 \\
\hline 11 & 60 & 61 \\
\hline 12 & 70 & 69 \\
\hline 13 & 64 & 63 \\
\hline 14 & 52 & 54 \\
\hline 15 & 47 & 45 \\
\hline 16 & 43 & 44 \\
\hline 17 & 40 & 40 \\
\hline 18 & 35 & 36 \\
\hline 19 & 31 & 31 \\
\hline 20 & 20 & 20 \\
\hline $\begin{array}{c}\text { Media } \\
\text { aritmética } \\
\text { (Temperatura) }\end{array}$ & 33,2 & 33,55 \\
\hline
\end{tabular}

Fuente: Elaboración propia de los autores. 
Tomando como base estos datos se comparó dos tratamientos mediante la prueba TStudent que permite utilizar una muestra de 30 datos en una distribución normal. Los valores del sensor DS18B20 y del termómetro deben seguir una distribución normal y se comprueba mediante una prueba de normalidad de Shapirowilk.

Como hipótesis para la prueba de distribución de T-Student son:

- Ho: Los datos siguen una distribución normal (hipótesis nula).

- Ha: Los datos no siguen una distribución normal (hipótesis alterna).

Tabla 5. Prueba de Normalidad Shapiro-Wilk

\begin{tabular}{|c|c|c|c|c|c|c|}
\hline \multirow{2}{*}{$\begin{array}{l}\text { Dispositivos } \\
\text { de medición }\end{array}$} & \multicolumn{3}{|c|}{ Kolmogorov-Smirnova } & \multicolumn{3}{|c|}{ Shapiro-Wilk } \\
\hline & Estadístico & Gl & Signi. & Estadístico & $\mathrm{Gl}$ & Signi. \\
\hline \multirow{2}{*}{$\begin{array}{l}\text { Valores de } \\
\text { temperatura }\end{array}$} & $\begin{array}{c}\text { Sensor } \\
\text { DS18B20 }\end{array}$ & 0,126 & 20 & 0,935 & 20 & 0,192 \\
\hline & $\begin{array}{c}\text { Termómetro } \\
\text { de Alcohol }\end{array}$ & 0,112 & 20 & 0,933 & 20 & 0,179 \\
\hline
\end{tabular}

Fuente: Elaboración propia de los autores.

La prueba de normalidad de Shapiro-Wilk puede brindar un valor de significancia (Signi) con valor de 0,192 con el sensor DS18B20 y 0,179 con el termómetro de alcohol, ambos tienen un nivel de significancia del 0,05 con lo cual se concluye que tienen una distribución normal.

Mediante la prueba de distribución T-Student de los 20 datos del sensor DS18B20 y el termómetro de alcohol se tiene un nivel de confianza del $95 \%$ con un valor de significancia del 0,05 para las hipótesis:

- Ho: La media de los datos captados por el sensor es igual a la media de datos de temperatura captados por el termómetro de alcohol (hipótesis nula)

- Ha: La media de los datos captados por el sensor no es igual a la media de los datos de temperatura captados por el termómetro de alcohol (hipótesis alterna)

Tabla 6. Prueba de Normalidad Shapiro-Wilk

\begin{tabular}{cccccc}
\hline Dispositivos de medición & N & Media & $\begin{array}{c}\text { Desviación } \\
\text { Típica }\end{array}$ & $\begin{array}{c}\text { Error } \\
\text { típico de } \\
\text { la media }\end{array}$ \\
\hline $\begin{array}{c}\text { Valores de } \\
\text { temperatura }\end{array}$ & $\begin{array}{c}\text { Sensor } \\
\text { DS18B20 }\end{array}$ & 20 & 33,20 & 17,999 & 20 \\
\hline
\end{tabular}


Vol. 4, N².2, p. 40-57, junio, 2021

$\begin{array}{cccc}\begin{array}{c}\text { Termómetro } \\ \text { de Alcohol }\end{array} & 20 & 33,55 & 17,834\end{array}$

Fuente: Elaboración propia de los autores.

\section{Manipulación directa de operario.}

Mediante el método de inducción se logró determinar el evento en que se encuentra el operario y el efecto en el proceso de pasteurización de leche.

En la tabla se observa los pasos y eventos en los que incide el operario que en un inicio se nota su presencia en los 5 pasos mientras que, después de la automatización del proceso tiene una mejoría de rendimiento de un $80 \%$ en vista del operario.

Tabla 7. Manipulación del operario vs automatización

\begin{tabular}{lcc}
\hline \multicolumn{1}{c}{ Secuencia del proceso } & $\begin{array}{c}\text { Manipulación } \\
\text { (Antes) }\end{array}$ & $\begin{array}{c}\text { Manipulación } \\
\text { (Después) }\end{array}$ \\
\hline $\begin{array}{l}\text { Ingreso de Materia } \\
\text { Prima(leche) }\end{array}$ & $\mathrm{Si}$ & $\mathrm{Si}$ \\
$\begin{array}{l}\text { Lectura de temperaturas } \\
\text { Homogenización }\end{array}$ & $\mathrm{Si}$ & $\mathrm{No}$ \\
Medición de tiempo & $\mathrm{Si}$ & $\mathrm{No}$ \\
Apertura de Válvulas/llaves de & $\mathrm{Si}$ & No \\
paso & $\mathrm{Si}$ & No \\
\hline \multicolumn{1}{c}{ Total Pasos } & 5 & 1 \\
\hline
\end{tabular}

Fuente: Elaboración propia de los autores.

La prueba de Levene, determinó una igualdad en la varianza con una significancia (Signi.) de 0,955 y este valor es mayor al 0,05 que se utilizará en cálculos. La prueba T-Student $(\mathrm{T})=-0,062$ y el valor crítico de dos colas (Sigin Bilateral) de $= \pm 0,951$ lo cual da una aceptación de hipótesis nula del tratamiento, se acepta el sensor DS18B20 para la adquisición de datos de temperatura.

Figura 6. Zona de Aceptación para la utilización del Sensor DS18B20.

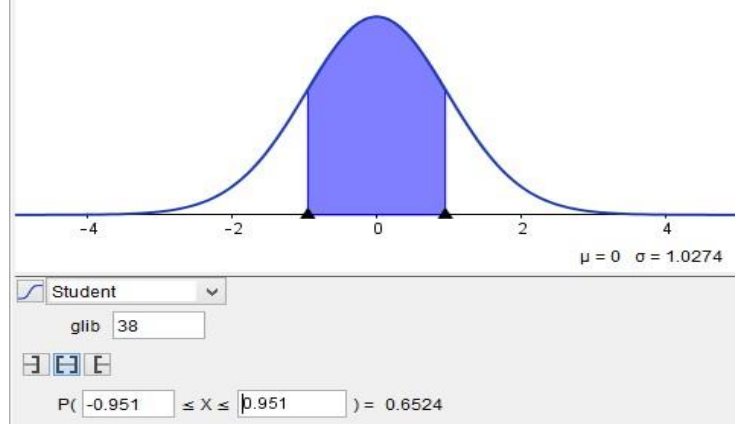

Fuente: Elaboración propia de los autores. 


\section{Homogenización}

El proceso de homogenización tiene como elementos un conjunto moto-reductor de marca Motor King Rigth sf7152 con aspas acopladas al eje del motor.

Figura 7. Aspas del pasteurizador

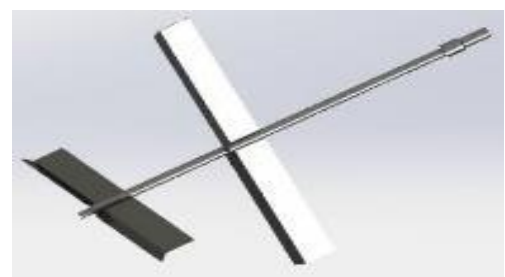

Fuente: Elaboración propia de los autores.

Este compuesto por un eje entre dos aspas y la marmita genera perdida de torque del motor reductor lo que provocó que el motor no arranque, se realiza un redondeo en el eje final de aspas para superar el factor de rozamiento entre las aspas, esto ayuda a un arranque similar con distintas cargas.

Mediante las pruebas 11 pruebas de arranque se determinó que se necesita 87 bits PWM para que el motor y las aspas comiencen a girar con carga dentro de la marmita, el motor trabaja en un ciclo de 34,5\% del valor máximo de PWM.

\section{Pruebas de la RNA (Red Neuronal Artificial) en MATLAB 2014}

\section{Plotperform}

Esta herramienta representa las actuaciones, pruebas y validación mediante el registro de entrenamiento optimizado MSE, se logró la reducción del índice de error con relación al número de iteraciones o número de épocas del sistema. Posterior a la determinación de los valores óptimos de respuesta para la RNA, surgieron 8 iteraciones haciendo uso del paquete RNA de MATLAB 2014.

Figura 8. Error cuadrático vs número de Iteraciones

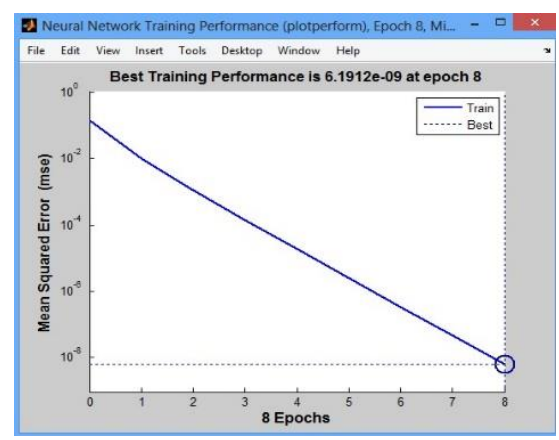

Fuente: Elaboración propia de los autores. 


\section{Plottrainstate}

Esta herramienta representa el estado de formación de un registro de vuelto por un tren de datos, indica la disminución de gradiente hasta una convergencia de la RNA, valores perdidos en validación y de no existencia en las 8 iteraciones. Se determina que la RNA entrega una salida con todos estos valores deseados de datos.

Figura 9. Estados de entrenamiento vs número de iteraciones.

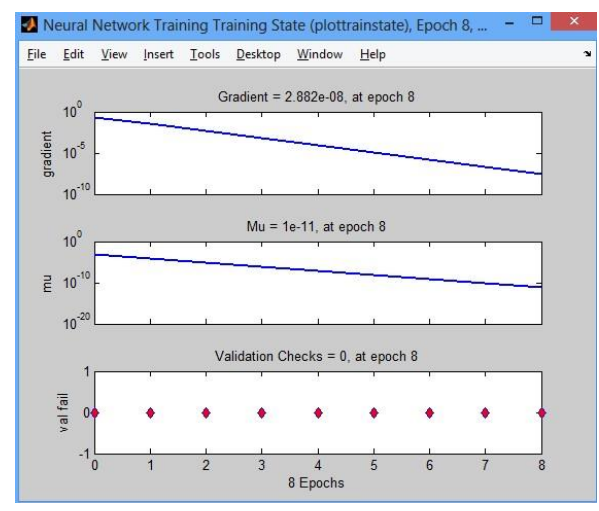

Fuente: Elaboración propia de los autores.

\section{Plotregression}

Esta herramienta permite visualizar una regresión lineal de los valores como entrada con relación a objetivos de salida, mediante este entrenamiento y la implementación de algoritmos nos permite observar el sistema linealizado y la determinación de no existencia de pérdidas de información durante el entrenamiento. Se logro entrenar todos los datos de una manera excelente.

Figura 10. Regresión de Datos de Entrenamiento

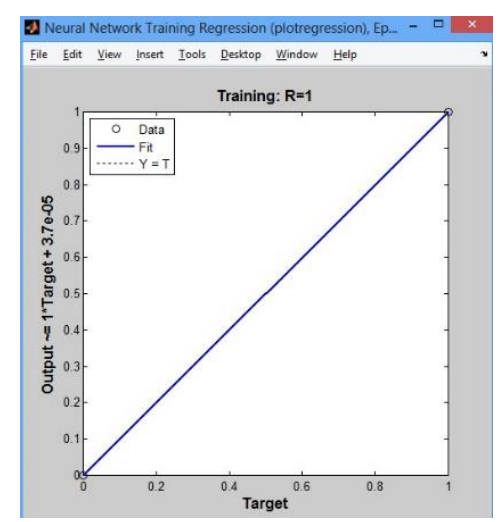

Fuente: Elaboración propia de los autores. 


\section{Conclusiones.}

- El proceso de pasteurización realizado con un sistema de control basado en una red neuronal permite la pasteurización con reducción de manipulación y el riesgo en un $80 \%$ del operario.

- La Red Neuronal Artificial (RNA) de tipo Feedforward contiene un algoritmo de backpropagation L-M y mitigación de error MSE, permite una convergencia de datos tanto de entrada como salida a su $100 \%$ con la aplicación de dos redes neuronales artificiales aplicadas a la subida y bajada asignadas al calentamiento y enfriamiento de la leche final.

- Mediante el uso del software Matlab 2014 y el hardware Arduino Mega 2560 se logró obtener un sistema neuro-controlador para temperatura con una respuesta optima y buena respuesta al proceso de pasteurización con buena tolerancia a fallas técnicas.

- La tarjeta Arduino Mega 2560 logró una lectura y escritura de gran cantidad de datos con una velocidad máxima de 115200 baudios.

\section{Recomendaciones.}

- Realizar una revisión previa de compatibilidad entre hardware y software de los componentes, así como sus hojas de datos para verificar voltajes máximos admitidos y evitar daños.

- Separar los circuitos de mando y potencia como protección ante un corto circuito, sobretensión o ruido en la etapa de mando y sistema neuro-controlador.

- Tomar en cuenta que los switches deben estar en modo activo, de igual manera los dispositivos de protección y alimentación.

\section{Referencias bibliográficas.}

ATRIA INNOVATION. (26 de 05 de 2021). ATRIAINNOVATION. Obtenido de https://www.atriainnovation.com/que-son-las-redes-neuronales-y-sus-funciones/

Gösta, B. (2003). Manual de industrias lácteas TetraPak Processing. Madrid, España: Madrid Vicente.

HaHn, N. (26 de 05 de 2014). GitHub. Obtenido de https://github.com/nhahn/gripitgood/blob/master/arduino_serial_control/pde/adi oes/adioes.pde

Hardware Libre. (26 de 05 de 2021). Hardwarelibre. Obtenido de https://www.hwlibre.com/irfz44n/

IMPROLAC. (26 de 05 de 2021). IMPROLAC. Obtenido de https://www.improlac.com/es/17/procesos-y-maquinas-tratamientos-termicosprocesos-uht.html 
INEN. (26 de 05 de 2021). INEN INSTITUTO ECUATORIANO DE NORMALIZACIÓN Norma Técnica Ecuatoriana NTE INEN 9:2010 Quinta Revisión. Obtenido de https://www.normalizacion.gob.ec/buzon/normas/9-5.pdf

INOXPA. (25 de 05 de 2021). INOXPA S.A.U. Obtenido de https://www.inoxpa.es/productos/equipos/tratamiento-termico/pasteurizador-htst

Interempresas. (15 de 10 de 2018). Canales sectoriales Interempresas. Obtenido de https://www.interempresas.net/Alimentaria/Articulos/227016-Pasteurizar-paragarantizar-la-seguridad-alimentaria.html

Mendieta Villamizar, J. C., Olarte Daza, M. A., \& González Acevedo, H. (2012). Diseño de un sistema de control inteligente para un pasteurizador tipo túnel. Fundación Dialnet, 81-88.

Ministerio de Agricultura y Ganadería. (12 de 11 de 2020). Ministerio de Agricultura y Ganadería. Obtenido de https://www.agricultura.gob.ec/ecuador-se-nutre-deleche-y-el-sector-lacteo-se-fortalece-con-apoyo-del-gobierno-nacional/

Palma Méndez, J., \& Márin Morales, R. (2011). Inteligencia Artificial: Métodos, técnicas y aplicaciones. Barcelona, España: McGraw-Hill.

Tipán, M., \& Flores, D. (2018). Diseño y construcción de un prototipo de pasteurizadora para el procesamiento de 50 litros de Leche/Hora. Quito, Pichincha, Ecuador: Universidad Politécnica Salesiana Sede Quito.

Tortora, G., Funke, B., \& Case, C. (2007). Introducción a la microbiología. Madrid: EDITORIAL MÉDICA PANAMERICANA. S.A.

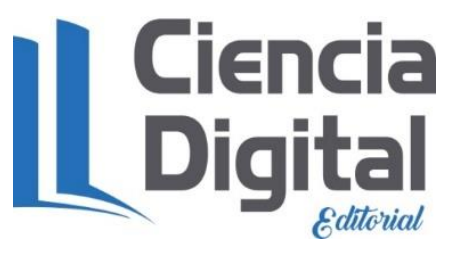




\section{PARA CITAR EL ARTÍCULO INDEXADO.}

Aldaz Vargas, A. A., Ocaña Pañora, L. S., Arias Guadalupe, J. I., \& Campos Castelo, J. P. (2021). Desarrollo de un sistema de control inteligente basado en redes neuronales aplicado al proceso de pasteurización de lácteos. ConcienciaDigital, 4(2.2), 40-57. https://doi.org/10.33262/concienciadigital.v4i2.2.1736

\section{Ciencia \\ LDigital}

El artículo que se publica es de exclusiva responsabilidad de los autores y no necesariamente reflejan el pensamiento de la Revista Conciencia Digital.

El artículo queda en propiedad de la revista y, por tanto, su publicación parcial y/o total en otro medio tiene que ser autorizado por el director de la Revista Conciencia Digital.

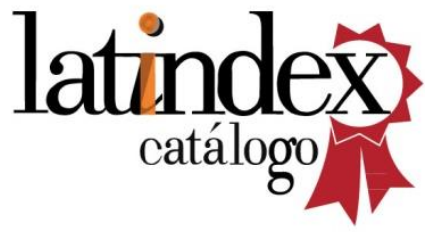

